\title{
Bywater syndrome is a rare complication of acute inflammatory myopathy: a case report and review of literatures
}

\begin{abstract}
The inflammatory myopathies include a group of diseases characterized by chronic muscle inflammation associated with weakness. There are three main types, polymyositis, inclusion body myositis and dermatomyositis. In here, we report a young male who developed a sudden attack of rhabdomyolysis with extreme elevation of creatine kinase (CK) and acute kidney injury. Muscle biopsy performed and revealed changes consistent with polymyositis. The patient condition improved dramatically to corticosteroid treatment.
\end{abstract}

Keywords: creatine kinase, acute interstial nephritis, acute renal failure, chronic renal failure, polymyositis, crush syndrome
Volume 8 Issue 5 - 2020

\author{
Ahmed AKL, ${ }^{1,2,3}$ Ayman Eissa,' Sayda Albluwi, ${ }^{3}$ \\ Mumtaz Ashour,' Souha Essa' \\ 'Dr. Soliman Fakeeh Hospital, Jeddah, KSA \\ ${ }^{2}$ Fakeeh college of Medical Sciences, Jeddah, KSA \\ ${ }^{3}$ Urology \& Nephrology Center, Mansoura University, Egypt
}

Correspondence: Ahmed AKL, MD, FACP, FASN, Associate Professor, Fakeeh College of Medical Sciences, Jeddah, KSA, DR Soliman Fakeeh Hospital, Jeddah, KSA, Urology \& Nephrology Center, Mansoura University, Egypt,

Email aiakl200I@yahoo.com

Received: September 08, 2020 | Published: September 25, 2020

\section{Introduction}

The crush syndrome was first described by the British physician Eric George Bywaters and others during world war II, London Blitz 1941 in 4 patients suffered severe rhabdomyolysis and acute kidney injury. ${ }^{1}$ The crush syndrome is a result of sudden crushing pressure to the muscle inducing severe ischemic injury and with the rapid release of that pressure lead to reperfusion injury. ${ }^{2}$ The pathophysiology is related to the release muscle breakdown products into the blood stream mainly myoglobin, phosphorus and potassium which represent products of rhabdomyolysis. ${ }^{2}$ A similar condition was observed in epileptic patients following a severe attack of seizure or due to direct muscle toxicity by the anti-epileptic drugs. The associated acute kidney injury cause is under investigated and mainly related to circulating nephrotoxic metabolites like myoglobin. ${ }^{3}$ Once the muscle cells injured they lost their ability to regulate sodium, water and calcium leading to rhabdomyolysis with absorption of water, sodium and release of potassium, myoglobin, phosphate, thromboplastin, creatine and creatine kinase. ${ }^{3}$ Barbiturate toxicity, poisoning with carbon monoxide, heat shock, severe hypokalaemia, and diabetic ketoacidosis represent just a few of the long list of causes that have been associated with nontraumatic muscle damage precipitating rhabdomyolysis. ${ }^{3}$ A few cases of patients with polymyositis developing acute kidney injury secondary to rhabdomyolysis been reported. ${ }^{4,5}$ Herein, we report a young man who presented with acute kidney injury secondary to acute polymyositis associated with rhabdomyolysis.

\section{Case report}

28-year-old male with brain anoxia at birth, history of epilepsy was on antiepileptic for three years till age of six. After a leisure trip to London with his family, he suddenly fall into ground with severe attack of seizure activity followed by myalgia and proximal muscle weakness. On transfer to our hospital, he was in shortness of breath, conscious, hypertensive, lung congestion and voiding less than $10 \mathrm{ml}$ of dark red urine per day. Investigations revealed normal sonographic kidneys, high serum creatinine $8.22 \mathrm{mg} / \mathrm{dl}$, sky-high serum creatine kinase (CK total) of $>420,700 \mathrm{u} / 1$ (normal 30-200u/l), hyponatremia $128 \mathrm{mmol} / \mathrm{l}$, hyperkalemia of $5.7 \mathrm{mmol} / \mathrm{l}$, hyperuricemia uric acid $9.0 \mathrm{mg} / \mathrm{dl}$, metabolic acidosis; serum bicarbonate was $15 \mathrm{mmol} / \mathrm{l}$, hyperphosphatemia $8 \mathrm{mg} / \mathrm{dl}$, hypocalcemia $6.2 \mathrm{mg} / \mathrm{dl}$ and positive myoglobin in urine. A case of rhabdomyolysis with acute kidney injury stage 3 was declared. Intra-venous sodium bicarbonate infusion with $77 \mathrm{meq}$ in one $1 / 2$ normal saline with flow rate of $1 \mathrm{ml} /$ $\mathrm{kg}$ /day was initiated and on next day supportive hemodialysis session was indicated for normalization of metabolic base, adjustment of fluid overload, removal of myoglobin toxic burden on nephrons. Febuxostat was initiated for control of hyperuricemia, sevelamer was added to control hyperphosphatemia, and hypocalcemia was left with no correction to avoid muscle necrosis. Daily, hemodialysis was done for 4 consecutive days.

Once urine output became satisfactory above $1200 \mathrm{ml} /$ day, with normal yellow colour and no detected myoglobin, hemodialysis stopped. In two week time the patient became polyuric with more than 6 liters per day (Figure 1), serum creatinine, BUN and creatine kinase dropped to normal levels with normalization of calcium, phosphorus, uric acid. However, once intravenous fluids stopped and urine output return to normal level, with improvement of serum creatine kinase, it start to increase again by around $200 \mathrm{mg} / \mathrm{dl}$ per day, with maintaining of normal renal function. Clinically, patient complained of severe right thigh pain, unilateral hypotonia related to right thigh muscles with unilateral oedema of whole right leg up to inguinal region, and inability to wake on his leg. Doppler us of arterial and venous system of the lower limbs was normal, EEG was done and reveal normal nerve conduction. Muscle biopsy was done under anesthesia, and pulse methylprednisolone was initiated $1000 \mathrm{mg}$ iv per day for 3 
days. On next day, a dramatic improvement of the patient clinical condition, he was walking with no pain in the hospital corridor. The muscles of the right thigh regained its normal tone and the oedema disappeared. The biopsy results came later and showed multifocal necrotic muscle fibers, myophagocytosis, up regulation of MHC-I in absence of inflammatory infiltrates which is consistent with picture of polymyositis. In view of this, patient was discharged on maintenance oral prednisolone $40 \mathrm{mg}$ daily with gradual tapering of the dose over 15 days. After two months, his serum creatinine $0.8 \mathrm{mg} / \mathrm{dl}$, normal serum creatine kinase (Figures $2 \& 3$ ).

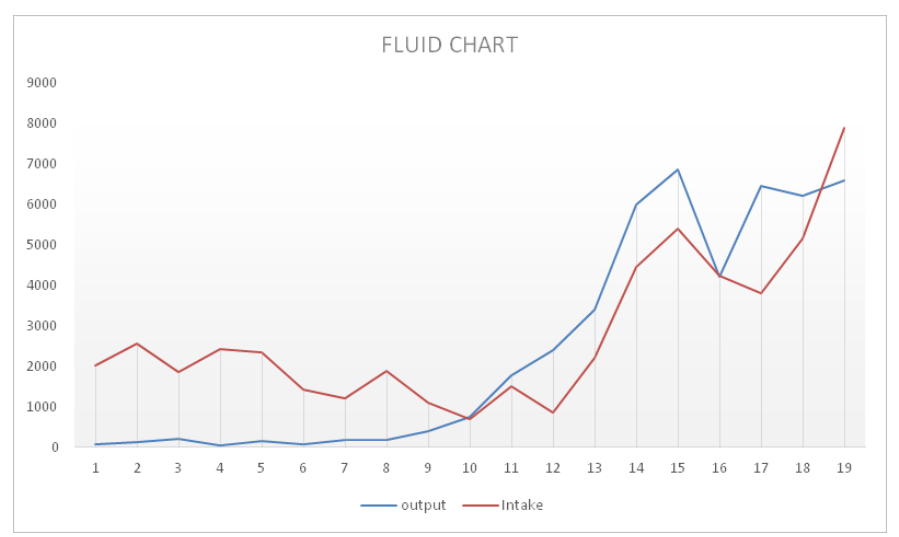

Figure I Daily monitoring of fluids intake versus urine output.

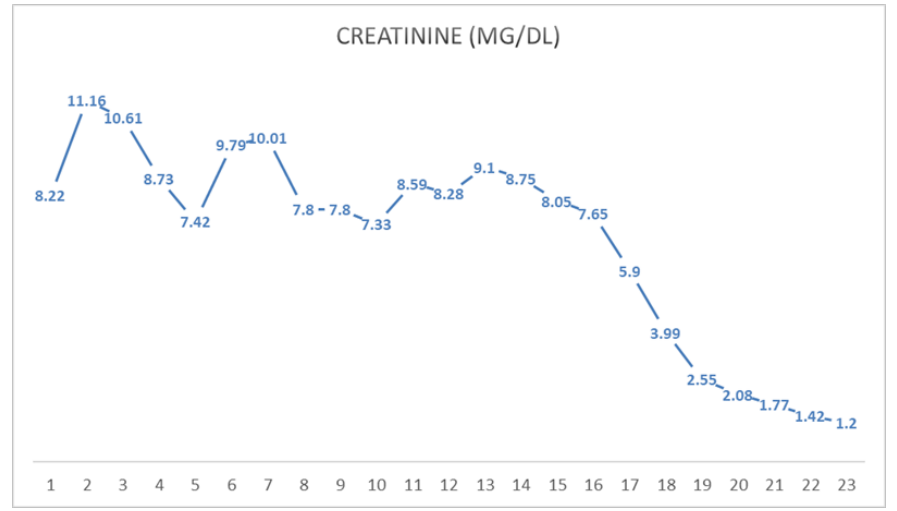

Figure 2 Serial daily serum creatinine.

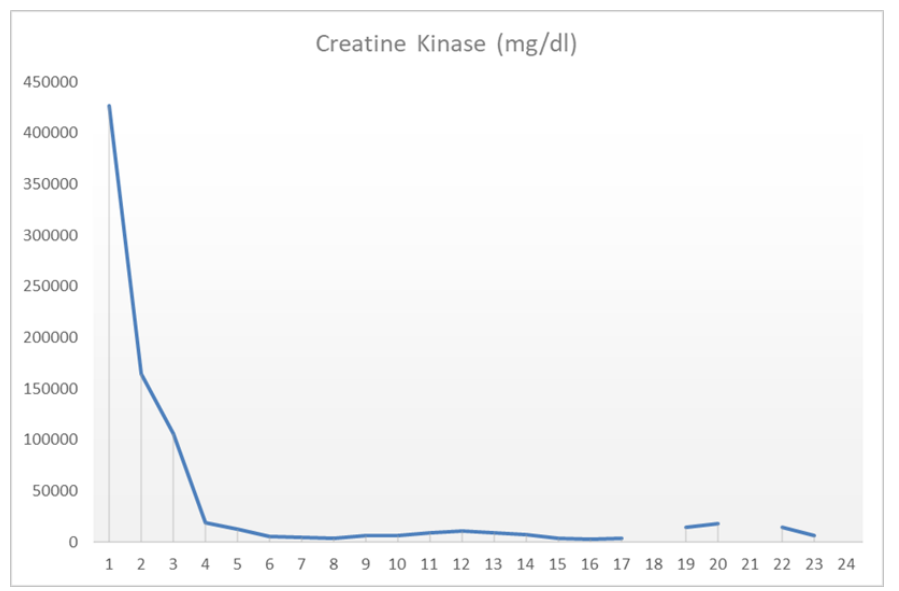

Figure 3 Serial daily serum creatine kinase total.

\section{Discussion}

Polymyositis is an inflammatory myopathy characterized by damage of skeletal muscle cells due to a perivascular hypoperfusion and/or direct lymphocytes infiltration of the muscle interstium. Polymyositis may develop at any age and in both genders. The majority of cases had background of autoimmune connectivetissue disorders and 10\% may have underlying malignancy. The identification of polymyositis relay on the clinical judgment, rise of serum creatinine kinase, electromyogram, and a diagnostic muscle biopsy. Acute extensive muscle injury lead to release of myoglobin that can be detected in urine. A few reports of acute polymyositis leading to acute kidney failure. ${ }^{4,5}$ The pathophysiology of kidney injury in rhabdomyolysis is under investigation however reduced glomerular filtration rate and tubular obstruction by myoglobin is identified. ${ }^{6}$ Myoglobin itself is relatively non-toxic, but becomes highly toxic in the presence of acidosis, dehydration, or both. ${ }^{7}$ In addition, release of iron compounds stimulates the formation of toxic free radicals leading to tubular epithelial damage. ${ }^{8}$ Myoglobin enhances vasoconstriction by inhibiting the production of endothelial relaxing factor. ${ }^{9}$ Due to its oxygen binding properties, myoglobin might theoretically interfere with oxygen availability in hypoxic areas of the kidney. It has therefore been proposed that renal damage could result from an imbalance between reduced oxygen supply and continued oxygen demand for inefficient transport. ${ }^{10}$

Once rhabdomyolysis is suspected or confirmed, good hydration and alkalinization of urine can protect from acute kidney injury if started early before oliguria. ${ }^{11} \mathrm{~A}$ intermittent period of renal replacement therapy is usually needed in case of unsatisfactory urine output to avoid complications of volume overload, electrolytes disturbance and to enhance recovery of kidney function. ${ }^{12}$ In case of delayed recovery, renal biopsy typically shows positive immunoperoxidase staining for myoglobin in case of rhabdomyolysis. ${ }^{13}$ In polymyositis, there have been associated mesangio-proliferative glomerulonephritis, with complement and immunoglobumin deposits in the mesangium. ${ }^{14}$ Our case demonstrates severe muscle lysis secondary to polymyositis leading to severe rhabdomyolysis, metabolic acidosis and hypovolaemia ended with acute kidney injury. Prompt diagnosis and treatment may avoid the need for prolonged haemodialysis. In conclusion, we report a rare case of crush syndrome complicated by local polymyositis after combined trauma and possible toxic effect from antiepileptic medications with good outcome after careful stepwise management.

\section{Acknowledgments}

We would like to thank our renal nurses and internal medicine nurses for their care of our patient through his admission time in the hospital.

\section{Conflicts of interest}

The author declares there is no conflict of interest.

\section{References}

1. Bywaters EGL, Beall D. Crush injuries with impairment of renal function. Br Med J. 1941;1:427-432.

2. Giannoglou GD, Chatzizisis YS, Misirli G. The syndrome of rhabdomyolysis: Pathophysiology and diagnosis. Eur $J$ Intern Med. 2007; 18:90. 
3. Bosch X, Poch E, Grau JM. Rhabdomyolysis and acute kidney injury. $N$ Engl J Med. 2009;361:62.

4. Sloan MF, Franks AJ, Exley KA, et al. Acute renal failure due to polymyositis. $\mathrm{Br}$ Med J. 1978;1:1457.

5. Ghosh AK, Sakhuja V Joshi K, Gupta KX, et al. Acute myositis complicated by myoglobinuric acute renal failure. $J$ Assoc Physicians India. 1993;41:453-454.

6. Cervellin G, Comelli I, Benatti M, et al. Non-traumatic rhabdomyolysis: Background, laboratory features, and acute clinical management. Clin Biochem. 2017;50(12):656-662.

7. Garcia G, Snider T, Feldman C, et al. Nephrotoxicity of myoglobin in the rat: relative importance of urine $\mathrm{pH}$ and prior dehydration (abstract) Kidney Int. 1981;19:200

8. Braun SR, Weiss FR, Keller Al, et al. Evaluation of the renal toxicity of haeme proteins and their derivatives: a role in the genesis of acute tubule necrosis. J Exp Med. 1970;131:443-460.
9. Edwards DH, Griffith TM, Ryley HC, et al. Haptoglobin-haemoglobin complex in human plasma inhibits endothelium-dependent relaxation. Evidence that endothelium derived relaxing factor acts as a local autacoid. Cardiovasc Res. 1986;20:549-556.

10. Knochel JP. Rhabdomyolysis and myoglobinuria. Annu Rev Med. 1982;33:435-443.

11. Ron D, Taiteldeum U, Michelson ML. Prevention of acute renal failure in traumatic rhabdomyolysis. Arch Intern Med. 1984; 144: 277-280.

12. Thomas MAB, Ibels LS. Rhabdomyolysis and acute renal failure. $N$ Engl J Med. 1974;291:807-811.

13. Gabon PA, Kaehny WD, Kelleher SP. The spectrum of rhabdomyolysis Medicine. 1982;61:141-152.

14. Dyck RF, Katz A, Gordon DA, et al. Glomerulonephritis associated with polymyositis. J Rheumatol. 1979;6:336-344. 